UDC 519.614

\title{
The rise in maximum determinant matrix complexity
}

\author{
Yu. N. Balonina, Research Fellow, orcid.org/0000-0002-5102-4139 \\ A. M. Sergeeva, PhD, Tech., Associate Professor, orcid.org/0000-0002-4788-9869 \\ A. A. Vostrikova, PhD, Tech., Associate Professor, orcid.org/0000-0002-8513-368, vostricov@mail.ru \\ a Saint-Petersburg State University of Aerospace Instrumentation, 67, B. Morskaya St., 190000, \\ Saint-Petersburg, Russian Federation
}

\begin{abstract}
Introduction: In spite of the apparent relation between maximum determinant matrices of even (Hadamard matrices) and odd orders, the latter have particularly complex patterns of repetitive elements. This is what makes them unique and attractive for various applications in visual data processing, coding and masking. Purpose: Developing the theory of maximum determinant matrices, with the focus on using computer-aided analysis, and calculating unique matrices with unique pattern structures in their portraits. Results: We have found some peculiarities of maximum determinant matrices, outlined their families related to Fermat numbers, demonstrated the complication of patterns in other matrices as their orders grow. The presumption about the increasing complexity of structures as the matrix orders grow is confirmed by a chain of matrix portraits we demonstrate. As applied to orthogonal Belevitch matrices, it follows that they cannot be found even in small orders such as 66 or 86.
\end{abstract}

Keywords - determinant, determinant maximum, quasi-orthogonal matrices, Hadamard matrices, Mersenne matrices, Fermat matrices, cyclic matrices.

For citation: Balonin Yu. N., Sergeev A. M., Vostrikov A. A. The rise in maximum determinant matrix complexity. Informatsionno upravliaiushchie sistemy [Information and Control Systems], 2021, no. 3, pp. 2-8. doi:10.31799/1684-8853-2021-3-2-8

\section{Introduction}

Mathematical objects, such as matrices with small number of their element values (levels), are corresponded by determinant-extreme solutions which find practical use in image processing and noise-immune coding problems [1, 2], such as impulse-noise-resistant coding of images transferred via telecommunication channels [3], formation of nested code structures based on Barker codes with improved auto-correlation functions [4], image transformation for better protection from unauthorized use [5], etc.

In spite of the successes in its practical use, the theory of building few-level matrices is far from being completed. The material it has accumulated has not been fully systematized yet. For example, it is well known that prime-number sizes of matrix blocks can be matched by simple symmetric or skew-symmetric patterns formed by a cyclic shift of the upper row of the block, moving the pushed-out elements into the beginning. Non-prime-number sizes can strike you with a great diversity of possible pattern structures, still poorly studied.

In some cases, the structure of matrices or their blocks stays cyclic regardless of whether their sizes are expressed by prime or composite numbers. Although the problem of searching for few-level matrices has been in discussion for about 150 years, starting from Silvester's works [6], the attention to the existence of universal structures (symmetric, cyclic, two circulant, negacyclic, edgeless or edged) for Hadamard matrices [7] was paid only recently
[8, 9]. Non-universal structures are studied even worse.

In other cases, splitting a matrix into one or two cyclic blocks is closely associated with the primeness of the number which expresses the matrix order. This seemingly natural rule has some exceptions [10] which ruin the integrity of the concept that a matrix order should be followed both numerical and pattern (pattern is ornament, structure) characteristics. The idea about a possible solution becomes rather ambiguous.

Maximum determinant matrices [11-13] are particularly unlucky, exactly because their properties, including pattern properties, are strongly pronounced. Researchers prefer to study isolated odd orders [14, 15], missing the overall picture, especially because they do not collate these orders with few-level orthogonal Hadamard and Belevitch matrices [7, 16, 17]. For example, Bush-type Hadamard matrices [10] are constrained by large square arrays of $1 \mathrm{~s}$ on their diagonal. Like Belevitch matrices with 0 s on their diagonal, they have even orders, but their pattern characteristics make them closer to the most complicated case, odd-order matrices.

In this work, we focus not on particular determinant-extremal matrices as mathematical objects difficult to calculate, and not even on their calculation methods, but on what unites them with and separates them from all the matrices from the vast Hadamard family, being figured out during the study of determinant properties. The purpose of this work is to present some common patterns in maximum determinant matrices, showing how uni- 
versal their structures are and how their complexity grows at higher orders.

\section{Simple and complex patterns of even-order matrices}

In order to study few-level matrix patterns, let us discuss two different cases which have influenced the matrix pattern theory.

In Fig. 1, you can see an Hadamard matrix with elements 1 and $\mathbf{- 1}$ shown on its portrait as white and dark cells, respectively. The matrix is of order 16 , consisting of a cyclic block and an edge. This is a classical combination, as it was in the 1960s when it was discovered that there are no edgeless cyclic matrices in orders higher than 4 .

The first row of the nested cyclic block of order $p=n-1$ can be easily found by an algorithm working in finite field arithmetics $G F(p)$. When M. Hall discovered a cyclic block of a composite size 15 [10, 18], it raised some perplexity because even when there is a field $G F\left(p^{m}\right)$, the internal block can be split into cyclic subblocks of size $p$. Later, two points were realized about such cases.

The first, almost apparent point is that 15 is a product of two close prime numbers 3 and 5 . The second, less known one can be checked by Fig. 1. In all "normal" matrices obtained by finite field algorithms, the elements placed symmetrically about the diagonal always differ in sign. Therefore, the edge is often inverted by multiplying the first row (or column) by -1 . But the above-mentioned matrix violates this rule, which means that a composite construction can always be recognized by its pattern. Mathematics leaves no room for arbitrariness and wanton deviation from its fundamental principles.

On evenly odd orders, instead of Hadamard matrices we search for Belevitch matrices which dif-

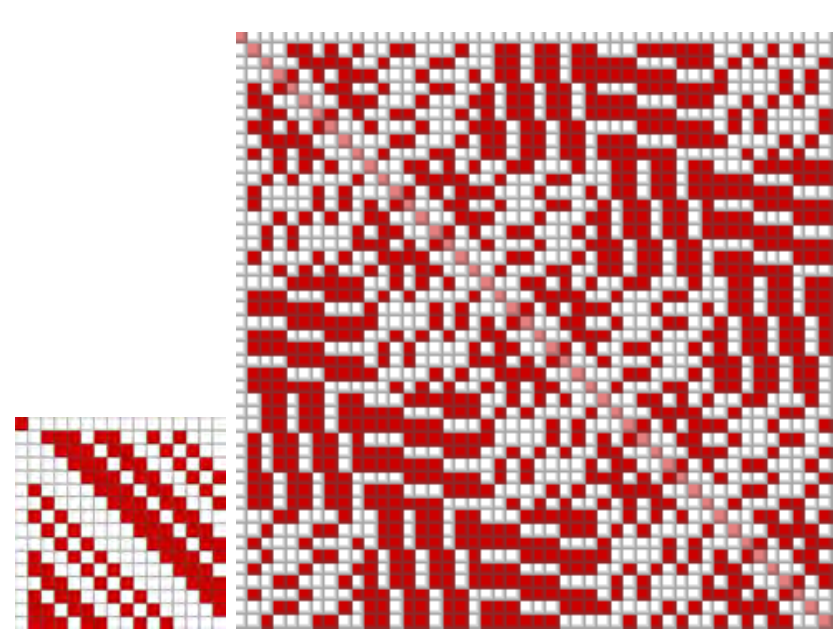

- Fig. 1. Portraits of Hadamard matrix of order 16 (left) and Belevitch matrix of order 46 (right) fer from Hadamard ones by a chain of 0s on their diagonal. In Fig. 1, they are highlighted in red. If the field $G F(p)$ exists, it exists for all prime $p$, and a Belevitch matrix is easy to find. Nevertheless, the $0 \mathrm{~s}$ on the diagonal are not $1 \mathrm{~s}$, and Belevitch matrices do not share the property of Hadamard matrices about having a certain universal structure formed at most by four cyclic blocks, a couple of negacyclic blocks or a couple of cyclic blocks and a double edge $[9,10]$. Apparently, there is a reason for Belevitch matrix patterns to become more complex, and this reason manifests itself in a very unexpected fashion.

In a chain of matrices of orders $6,26,46,66,86$, $106,126 . .$. , with an interval 20 , the first matrix of order 6 has the above-mentioned simple structure which corresponds to the prime number $p=5$. In fields $G F(25), G F(125)$, the internal block consists of cyclic subblocks, therefore the matrix orders do not cause any size problems.

The order $46=5 \times 9+1$ is a different matter. It is easy to see that the edge in this decomposition corresponds to 1 , while the numbers 3 and 5 form a close couple ( 9 is a prime number power). Nevertheless, two blocks out of 5 in this decomposition partially lose their cyclic structure: they consist of $1,-1,-1$ sequences cyclically shifted by three. The solution is very uncommon for Hadamard matrices.

But really interesting is that this trick does not work for matrices of adjacent orders 66 and 86 . However, the precondition for their existence is met, as the values $p=n-1$ in both cases can be split into two squares: $65=16+49,85=36+49$.

Note that the simplest one- or two-blocked edgeless or double-edged structures have been verified in a computer experiment (in [8] and other works to be published). There are two assumptions left: either Belevitch matrices of order 66 and 86 differ from each other, having, in respect to the matrix of order 46, a new unpredictable pattern element, or, which is less probable, the demand for such an element contradicts the symmetry, and these matrices do not exist. Both these cases are probable, having some strict logic behind them, which is difficult to check at the current level of computing power.

We do not exhaustively understand the nature of these maximum determinant matrices on the set of orthogonal matrices of the same order. But we do know something about their pattern invariants.

\section{Simple and complex patterns in odd-order matrices}

Maximum determinant matrices are square real-number matrices of order $n$ with an extremal (largest in magnitude) determinant among the matrices with elements not exceeding 1 in magnitude. 
As a matter of fact, at the moment there is no theory of such matrices. We will try to explain why.

Among them, there are few odd-order matrices with a simple structure. However, there is a presumption for orders equal to prime Fermat numbers, which is crucial for this topic. It is systemically important, so we will discuss it first.

Prime Fermat numbers are classical objects in the theory of numbers. The first five of them are $F_{k}=3,5,17,257,65537$. Back in 1796, C. F. Gauss, in his student days, discovered an unexpected relation between them and geometrical figures. He inscribed a regular 17-gon in a circle and proved a more general proposition that if a regular polygon has a number of sides equal to a prime Fermat number, it can be constructed by means of compasses and ruler.

Conjecture. Fermat matrices of the form

$$
\mathbf{F}=\left(\begin{array}{ccc}
-1 & \mathbf{e}^{\mathrm{T}} & \mathbf{e}^{\mathrm{T}} \\
\mathbf{e} & \mathbf{A} & \mathbf{B} \\
\mathbf{e} & \mathbf{B}^{\mathrm{T}} & -\mathbf{A}^{\mathrm{T}}
\end{array}\right)
$$

built on a pair of cyclic blocks $\mathbf{A}$ and $\mathbf{B}$ with an edge vector $\mathbf{e}$, of orders equal to prime numbers $n=F_{k}=3,5,17,257,65537, \ldots$ are maximum determinant matrices. $F_{k-1} /\left(2 F_{k}-1\right)^{1 / 2} \times \mathbf{B}$, $\mathbf{B}=(n-1)^{(n-1) / 2}(2 n-1)^{1 / 2}$ is Barba's upper-bound estimation of the odd-order matrix determinant [19].

For matrices of the first three orders whose portraits are shown in Fig. 2, these patterns are easy to find with the use of a computer.

Gauss's theorem suggests that due to deep interdisciplinary links in mathematics, these matrices are of special importance, not less than the 17-gon had for Gauss. His discovery is highly valued by geometricians and other specialists who want to see the integrity of a knowledge area artificially divided for the convenience of study. Here we add a new abstract example to it.
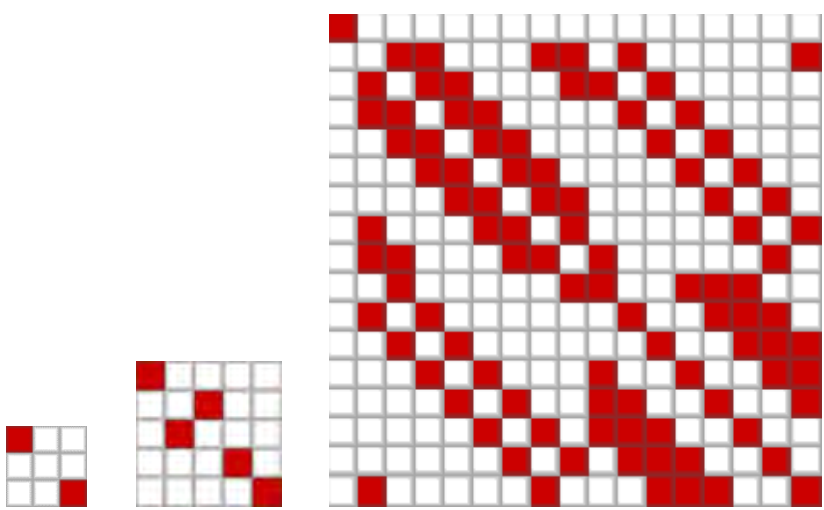

Fig. 2. Portraits of two circulant Fermat matrices of orders 3,5 , and 17
Note that if we discard the edge, the core of such matrices will be an Hadamard matrix. Regular Hadamard matrices of orders 8, 32, 64 similar to the above-mentioned matrices and their other versions easily found in a two circulant form by doubling Golay sequences [10] given in Table do not have this feature. We suppose that any other Hadamard matrices with any patterns in their portraits do not have it either. Therefore, the position taken by the Fermat matrices discussed above is absolutely unique. They are some kind of ideal "correct" matrices. They did not receive much attention so far because nobody thought about patterns. The competition was for uniqueness, not for residual universality.

It is easy to guess that the next to come are Mersenne numbers in the form $n=2^{k}-1$. Among them, there are many prime numbers, including 15 discussed above. However, they give us only two maximum determinant matrices with a prime structure: of orders 3 and 7. Their portraits are shown in Fig. 3. As we can see, they can be considered two circulant (splitting the core into 4 squares) or edged monocyclic ones. They have a maximum determinant, and here is the end of the Mersennian chain. It lacks the quality of the Fermat matrix chain.

Maximum determinant matrices are not orthogonal. Unlike Hadamard matrices, they are not constricted by the orthogonality condition, but they coincide with Hadamard matrices on evenly even orders. Therefore, Ryser's circulant structure border [20] which is set for Hadamard matrices at a

Golay sequences

\begin{tabular}{|c|l|}
\hline $\begin{array}{c}\text { Length } \\
n\end{array}$ & \multicolumn{1}{c|}{ Golay sequences } \\
\hline 2 & $\mathbf{a}=[1,1], \mathrm{b}=[1,-1]$ \\
\hline 10 & $\begin{array}{l}\mathbf{a}=[1,1,-1,1,-1,1,-1,-1,1,1] \\
\mathbf{b}=[1,1,-1,1,1,1,1,1,-1,-1]\end{array}$ \\
\hline 26 & $\begin{array}{l}\mathbf{a}=[1,1,1,1,-1,1,1,-1,-1,1,-1,1,-1,1,-1,-1, \\
1,-1,1,1,1,-1,-1,1,1,1] \\
\mathbf{b}=[1,1,1,1,-1,1,1,-1,-1,1,-1,1,1,1,1,1,-1, \\
1,-1,-1,-1,1,1,-1,-1,-1]\end{array}$ \\
\hline
\end{tabular}

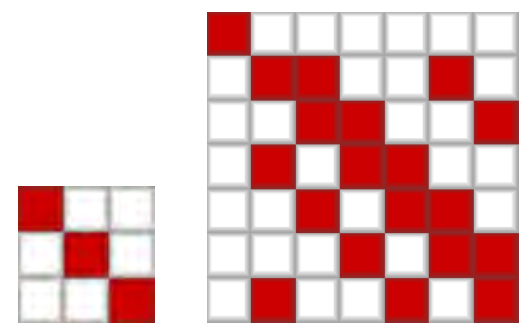

- Fig. 3. Portraits of Mersenne matrices of orders 3 and 7 
very low level (order 4), in this case is expanded up to three matrices of orders 3,5 , and 13 . You can see their portraits in Fig. 4. Note that 5 and 13 are related to the key lengths of Golay sequences.

Now, it would be logical to presume that, by analogy with Gauss's theorem interpreted in an abstract way, there can be no simple pattern maximum determinant matrices.

There are certain starting orders in which you can meet some simple pattern matrices without violating the general principle. Besides, Barba [21] in his upper-bound estimation of maximum determinant noticed two chains of rapidly growing orders, so called "Barba's whiskers" in which integer matrices follow these integer border points (Gauss's points) by determinant.

Note that Fermat matrices do not go as far as this border. They were overlooked by researchers who focused on optimistic estimation to the detriment of common sense which suggested focusing on resolvable orders.

The orders found by Barba are not resolvable. The most well-known solution is the one of order 25 proposed by Raghavarao in [22]. It stimulated Andre Brouwer [23] to prove a theorem that there are no explicit solutions for them (we also think so). Brouwer proved that for a half of all orders a solu-
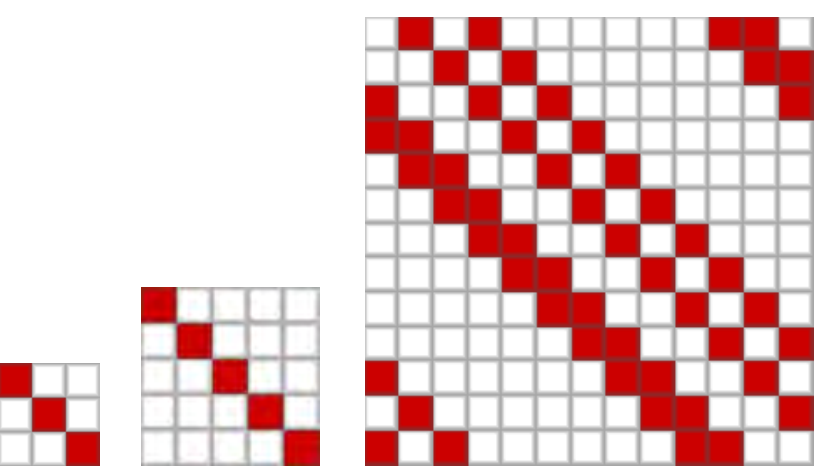

Fig. 4. Portraits of three cyclic versions of maximum determinant matrices tion would consist of blocks with Hadamard matrix properties and an edge. Since the structure of these Hadamard matrices is not specified, this path leads to nowhere.

Still it is interesting to compare this path with Fermat matrices which exceed all such matrices. In order to complete our review of odd-order maximum determinant matrices, let us present the matrices of orders 9,11 , and 15 which were omitted so far. You can see their patterns in Fig. 5.

What we need to do now is to find the fundamental difference between these three matrix patterns. These matrices are unique, although all the three are constructed by the use of the same building block: Mersenne matrix of order 3 with a usual or inverted (in the third solution) diagonal when it forms a new block out of 1 or -1 . Another difference is the block edge of different width, with lengthwise elements found in the Belevitch matrix of order 46.

It is difficult to find solutions, but software with the self-describing name "Kaleidoscope" can synthesize them, dealing with a Mersenne matrix as if it were an Hadamard one. You still should remember how few good solutions can be found among Mersenne matrices.

Now let us consider matrices of the next odd orders, namely 19, 21, and 23. Their patterns are shown in Fig. 6.

These matrices could be expected to be simpler because, unlike the previous ones, they all are

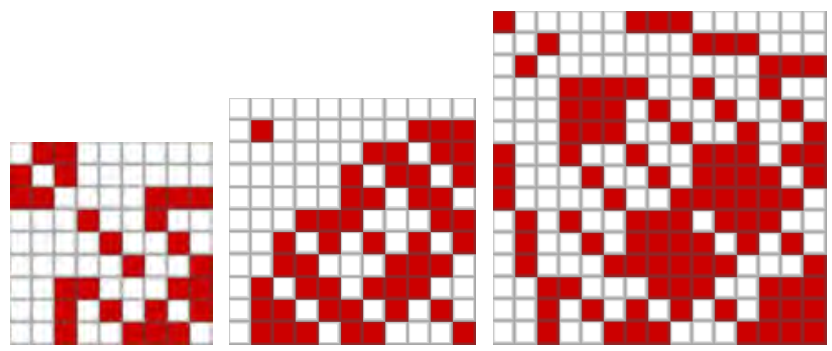

- Fig. 5. Patterns of matrices of orders 9, 11, and 15
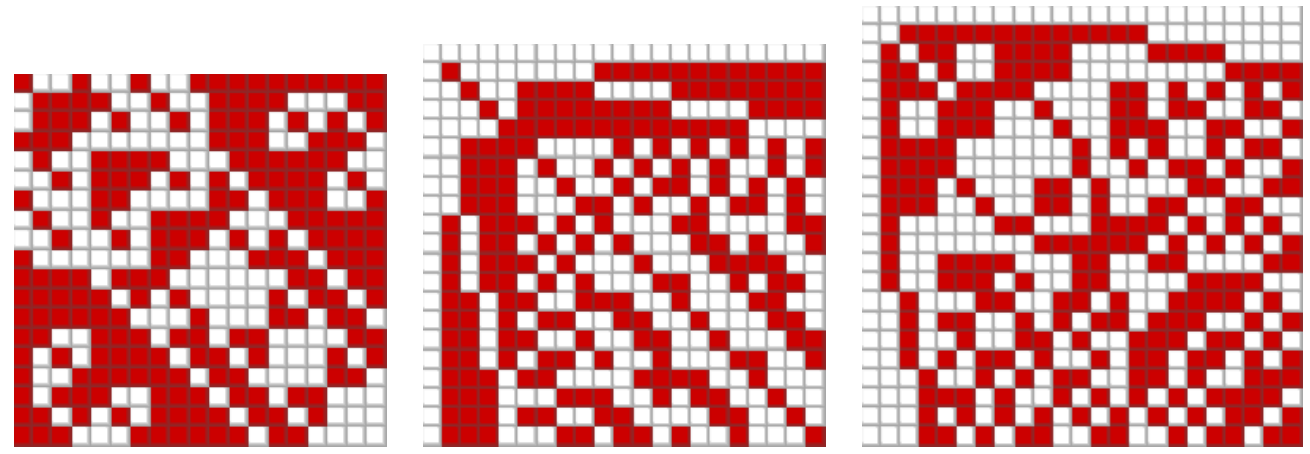

- Fig. 6. Patterns of matrices of orders 19, 21, and 23 
of prime orders. In their structure, we still find similar low-order blocks. However, in order 19, the starting block of size $10 \times 10$ containing 9 Mersenne matrices with a usual or inverted edge also contains an extra inversion of the first and then of every third element, both vertically and horizontally. Definitely, it is a new element, never met before. Possibly, this is the last symmetric matrix out of the considered ones. The matrix of order 21 is constructed from blocks of a higher order 4 , and it is not generally symmetric. The matrix of order 23 is more complicated. We cannot be definite about the order of its blocks, or where its block edge is, or what can be considered as its core.

\section{Complex patterns of matrices of Barba's orders}

The basic inequality for determinants of matrices $\mathbf{A}$ of odd orders was suggested by Guido Barba [21] in the following form:

$$
|\operatorname{det}(\mathbf{A})|^{2} \leq \operatorname{det}((n-1) \mathbf{I}+\mathbf{J})=(n-1)^{n-1}(2 n-1),
$$

where $\mathbf{I}$ is an identity matrix, and $\mathbf{J}$ is a matrix of $1 \mathrm{~s}$.

Since the optimal matrix and its determinant are integer, the maximum is achievable in orders for which $2 n-1$ is a square of an integer. Otherwise, square rooting the expression on the right will give us an irrational number a fortiori greater than the determinant. Hence, the border is achievable in a sequence of orders described by a sum of squares of two close numbers $n=a^{2}+(a+1)^{2}$ :

5, 13, 25 (Raghavarao),

41 (Bridges, Hall and Hayden),

61 (Brouwer),

85 (approximation by Solomon and Orrick),

113 (Brouwer), etc.

The names in brackets belong to the authors who were first to find matrices of the respective orders. These are matrices from two families, and their portraits are shown in Fig. 7, because the pattern complexity alternates every other order and matrices of orders 41,85 , etc. are much more difficult to find. Originally, these matrices were described by Raghavarao [22], and Andre Brouwer noticed that they were vitrages, i.e. matrices obtained by inserting blocks into Hadamard matrices. The principle of growing pattern complexity is true for them, too. The algorithm for constructing Barba's border matrices was described by Andre Brouwer and his followers [24] only roughly; to apply it, you need an Hadamard matrix, but the way to construct Hadamard matrices is not fully known. We also do not know Barba matrices of orders 145, 181, 221, $265,313,365,421,481,545,613,685$, etc.
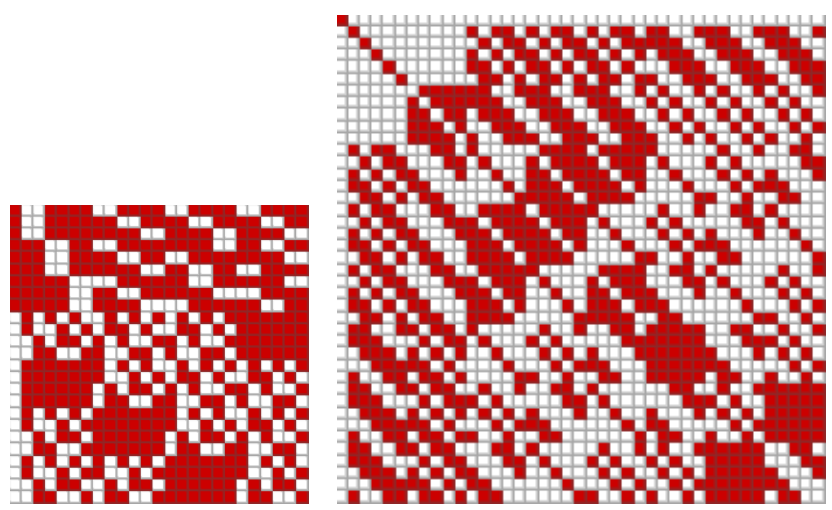

- Fig. 7. Portraits of Barba matrices of orders 25 and 41

Thus, in each considered order, we can see an original element as a pattern part, and all these elements are unique. Our presumption (conjecture) is that this is a key feature of odd-order maximum determinant matrices everywhere outside the orders equal to Fermat numbers. This point dramatically reduces the chances to calculate them, lending weight to the alternative proposals about searching for matrices which achieve the maximum determinant on a preset structure borrowed from the optimal solutions for Fermat matrices [25, 26].

\section{Pattern symmetry border}

The presence of a symmetry border can be traced on two matrices of low even orders 22 and 34. The best pattern solution for orders 22,34 and similar to them is based on the fact that $n-1$ is not resoluble into a sum of two squares (except order 58), which gives us double-edged two circulant matrices. You can see the portraits of maximum determinant matrices of orders 22 and 34 in Fig. 8.

This structure is universal for Hadamard matrices. The symmetry border for edgeless two circulant

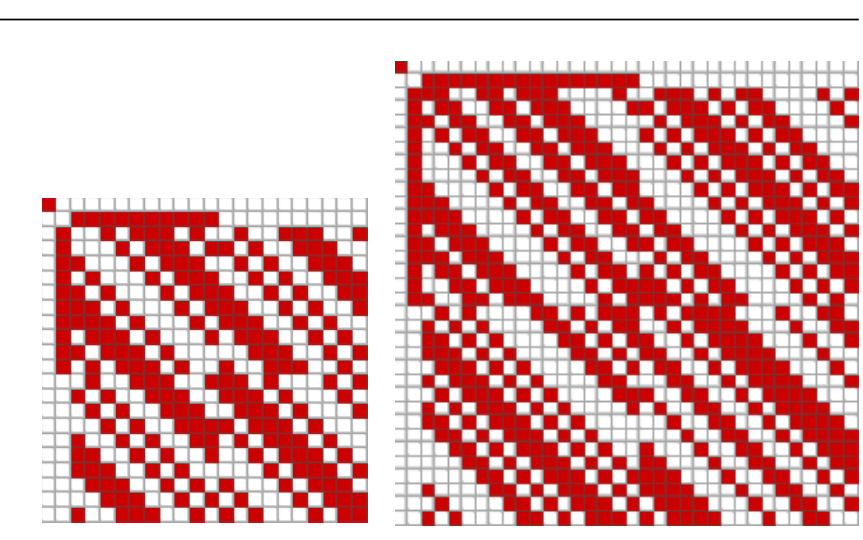

- Fig. 8. Portraits of double-edged two circulant matrices of orders 22 and 34 
matrices in Hadamard matrix orders was found in the critical order 32 [8]. In maximum determinant matrices, this border is shifted much farther for Barba's double orders, but is also limited (most likely, by order 82). The matrix of order 22 is unique in being the only doubly symmetric edged two circulant matrix whose both blocks $\mathbf{A}$ и $\mathbf{B}$ are symmetric.

\section{Conclusion}

The efficiency of an algorithm depends on the representation complexity of the mathematical object in the problem to be solved. The same object, for example a number, can be written either as a Roman number or in a positional notation. The latter provides simpler rules which, in old days, fostered the ideas of al-Khwarizmi whose name and whose book gave life to the words "algorithm" and "algebra".

Such an obvious example of advantageous object representation stimulated the research of computational complexity conducted by some famous logicians of the 20th century: Kurt Gödel, Andrey

\section{References}

1. Ahmed N., Rao R. Orthogonal Transforms for Digital Signal Processing. Berlin-Heidelberg-New York, Springer-Verlag, 1975. $263 \mathrm{p}$.

2. Wang R. Introduction to Orthogonal Transforms with Applications in Data Processing and Analysis. Cambridge University Press, 2010. 504 p.

3. Mironovskii L. A., Slaev V. A. The strip method of noise-immune image transformation. Measurement Techniques, 2006, no. 8, pp. 6-12.

4. Sergeev M. B., Nenashev V. A., Sergeev A. M. Nested code sequences of Barker - Mersenne - Raghavarao. Informatsionno-upravliaiushchie sistemy [Information and Control Systems], 2019, no. 3, pp. 71-81 (In Russian). doi:10.31799/1684-8853-2019-3-71-81

5. Vostrikov A. A., Sergeev M. B., Balonin N. A., Sergeev A. M. Use of symmetric Hadamard and Mersenne matrices in digital image processing. In: Procedia Computer Science, 2018. Pp. 1054-1061. doi:10.1016/j. procS.2018.08.042

6. Sylvester J. J. Thoughts on inverse orthogonal matrices, simultaneous sign successions, and tessellated pavements in two or more colours, with applications to Newton's rule, ornamental tile-work, and the theory of numbers. London Edinburgh and Dublin Philos. Mag. and J. Sci., 1867, vol. 34, pp. 461-475.

7. Hadamard J. Résolution d'une question relative aux d terminants. Bulletin des Sciences Mathématiques, 1893, vol. 17, pp. 240-246 (In French).

8. Seberry J., Yamada M. Hadamard Matrices: Constructions using Number Theory and Linear Algebra. John Wiley \& Sons, 2020. 352 p.
Markov, Pyotr Novikov, Alan Turing, and Alonzo Church.

Our current research is devoted to representation of maximum determinant matrices: this is the pattern whose properties are discussed in this article. We have specified several primary families, including matrices of orders equal to Mersenne or Fermat numbers, and made a presumption about the leading role of Fermat matrices based on their comparison with objects from a different field of mathematics studied by Gauss. Fermat matrices of high and super high orders can become another example of a discovery "with the point of your pen" due to finding a relation between objects of very different nature from different mathematical fields.

\section{Financial support}

The paper was prepared with the financial support of the Ministry of Science and Higher Education of the Russian Federation, grant agreement No. FSRF-2020-0004.

9. Djokovic D. Z. Some new symmetric Hadamard ma trices. arXiv:2101.05429 [math.CO]. Available at: https://arxiv.org/abs/2101.05429, free access (accessed 14 January 2021).

10. Handbook of Combinatorial Designs. Second Edition (Discrete Mathematics and its Applications). Charles J. Colbourn and Jeffrey H. Dinitz (Eds.). Chapman and Hall/CRC, 2006. 1000 p.

11. Osborn J. H. The Hadamard Maximal Determinant Problem. Honours thesis. University of Melbourne, 2002. 144 p. [Electronic resource]. Available at: http:// maths-people.anu.edu.au/ osborn/publications/ pubsall.html, free access (accessed 22 November 2020).

12. Neubauer M. G., and Radcliffe A. J. The maximum determinant of +1 matrices. Linear Algebra and its Applications, 1997, vol. 257, pp. 289-306.

13. Seberry J., Xia T., Koukouvinos C., Mitrouli M. The maximal determinant and subdeterminants of \pm 1 matrices. Linear Algebra and its Applications, 2003, vol. 373, pp. 297-310. Combinatorial Matrix Theory Conference (Pohang, 2002). doi:10.1016/S00243795(03)00584-6

14. Orrick W. P. The maximal $\{-1,1\}$-determinant of order 15. Metrika, 2005, no. 62, pp. 195-219.

15. Brent R. P., Orrick W. P., Osborn J. H., Zimmermann P. Maximal determinants and saturated D-optimal designs of orders 19 and 37 (preprint) [Electronic resourse]. Available at: http://arxiv.org/abs/1112.4160 (accessed 05 March 2020).

16. Seberry J., Yamada M. More on Maximal Determinant Matrices. In: Hadamard Matrices: Constructions using Number Theory and Linear Algebra. John Wiley \& Sons, 2020. Pp. 245-249. 
17. Balonin N. A., Đoković D. Ž. Conference matrices from Legendre C-pairs. Informatsionno-upravliaiushchie sistemy [Information and Control Systems], 2020, no. 4, pp. 2-10. doi:10.31799/1684-8853-2020-4-2-10

18. Hall M. A survey of difference sets. Proc Amer. Math. Soc., 1956, vol. 7, pp. 975-986.

19. Balonin N. A., Sergeev M. B., Vostrikov A. A. Prime Fermat numbers and maximum determinant matrix conjecture. Informatsionno-upravliaiushchie sistemy [Information and Control Systems], 2020, no. 2, pp. 2-9. doi:10.31799/1684-8853-2020-2-2-9

20. Balonin Yu. N., Sergeev A. M. Two-circulant Hadamard matrices, weighing matrices, and Ryser's conjecture. Informatsionno-upravliaiushchie sistemy [Information and Control Systems], 2018, no. 3, pp. 2-9. doi:10.15217/issn1684-8853.2018.3.2

21. Barba G. Intorno al teorema di Hadamard sui determinanti a valore Massimo. Giorn. Mat. Battaglini, 1933, vol. 71, pp. 70-86 (In Italian).
22. Raghavarao D. Some optimum weighing designs. Ann. Math. Statist., 1959, no. 30, pp. 295-303.

23. Brouwer A. E. An infinite series of symmetric designs. Math. Centrum Amsterdam Report ZW 202/83, 1983, pp. 1-5.

24. Popescu O., Rose C., Popescu D. Maximizing the determinant for a special class of block-partitioned matrices. Mathematical Problems in Engineering, 2004, no. 1 , pp. $49-61$.

25. Balonin N. A., Sergeev M. B., Seberry J., Sinitsyna O. I. Circles on lattices and Hadamard matrices. Informatsionno-upravliaiushchie sistemy [Information and Control Systems], 2019, no. 3, pp. 2-9 (In Russian). doi:10.31799/1684-8853-2019-3-2-9

26. Balonin N. A., Sergeev M. B., Seberry J., Sinitsyna O. I. Circles on lattices, and maximum determinant matrices. Informatsionno-upravliaiushchie sistemy [Information and Control Systems], 2020, no. 6, pp. 2-11 (In Russian). doi:10.31799/1684-8853-2020-6-2-11

УДК 519.614

doi:10.31799/1684-8853-2021-3-2-8

\section{Рост сложности матриц максимума детерминанта}

Ю. Н. Балонин ${ }^{\mathrm{a}}$, научный сотрудник, orcid.org/0000-0002-5102-4139

А. М. Сергеев ${ }^{\text {a }, ~ к а н д . ~ т е х н . ~ н а у к, ~ д о ц е н т, ~ o r c i d . o r g / 0000-0002-4788-9869 ~}$

А. А. Востриков ${ }^{a}$, канд. техн. наук, доцент, orcid.org/0000-0002-8513-368, vostricov@mail.ru

а Санкт-Петербургский государственный университет аэрокосмического приборостроения, Б. Морская ул., 67,

Санкт-Петербург, 190000, РФ

Введение: несмотря на очевидную связь матриц максимума детерминанта четных (матриц Адамара) и нечетных порядков, последние имеют особенно сложные структуры, что делает их уникальными и привлекательными для различных применений в обработке визуальной информации, ее кодировании и маскировании. Цель: развитие теории матриц максимального детерминанта с опорой на использование компьютерного анализа, а также вычисление новых матриц с уникальными структурами орнамента их портретов. Результаты: выявлены особенности матриц максимального детерминанта, выделены их семейства на порядках, связанных с числами Ферма, показано усложнение структурных закономерностей в матрицах других порядков по мере их роста. Предположение об усложнении структур по мере роста порядков матриц подтверждается цепочкой демонстрируемых матричных портретов. Применительно к ортогональным матрицам Белевича следует, что они не могут быть найдены даже на таких небольших порядках, как 66 или 86.

Ключевые слова - детерминант, максимум детерминанта, квазиортогональные матрицы, матрицы Адамара, матрицы Мерсенна, матрицы Ферма, циклические матрицы.

Для цитирования: Balonin Yu. N., Sergeev A. M., Vostrikov A. A. The rise in maximum determinant matrix complexity. Информационно-управляющие системы, 2021, № 3, с. 2-8. doi:10.31799/1684-8853-2021-3-2-8

For citation: Balonin Yu. N., Sergeev A. M., Vostrikov A. A. The rise in maximum determinant matrix complexity. Informatsionnoupravliaiushchie sistemy [Information and Control Systems], 2021, no. 3, pp. 2-8. doi:10.31799/1684-8853-2021-3-2-8

\section{Дополнение к статье}

Sivak S. A., Royak M. E., Stupakov I. M., Voznuk E. S., Aleksashin A. S. The implementation of the boundary element method to the Helmholtz equation of acoustics, опубликованной в № 2 за 2021 год:

\section{Financial support}

The reported study was funded by RFBR, project no. 19-31-90052.

\section{Финансовая поддержка}

Исследование выполнено при финансовой поддержке РФФИ в рамках научного проекта № 19-3190052. 